\title{
74. THE USE OF THE HALPHEN-GORYACHEV METHOD IN THE STUDY OF THE EVOLUTION OF THE ORBITS OF THE QUADRANTID AND $\delta$ AQUARID METEOR STREAMS
}

\author{
A. F. ZAUSAEV \\ Institute of Astrophysics, Dushanbe, U.S.S.R.
}

The Halphen-Goryachev method has been used for estimating the elements of the orbits of the Quadrantid and $\delta$ Aquarid meteor streams $4000 \mathrm{yr}$ back from the epoch of osculation. Three meteor orbits were selected for each stream: the mean orbit, based on data by Shelton (1965) and by Jacchia and Whipple (1961), and also two variants. Two cases were discussed: in the first case only the perturbations by Jupiter were taken into account, and in the second those by Jupiter and Saturn. Designating the initial epoch of osculation as the year zero, we compared our results for the Quadrantids with those by Hamid and Youssef (1963) at the final epoch (the year -4000). The disagreements in the elements $i, \omega$, and $\Omega$ were $3^{\circ}, 8^{\circ}$, and $1^{\circ}$, respectively (in absolute value). In comparing the mean orbits of the two streams it was noticed that between the years -800 and -900 , and also between -2300 and -2400 , the eccentricities and inclinations to the ecliptic were practically identical. However, the minimum differences in $\Omega$, and $\omega$ were $174^{\circ}$ and $153^{\circ}$ between -800 and -900 , and $15^{\circ}$ and $30^{\circ}$ between -2300 and -2400 . Consequently, if only secular perturbations are taken into account we cannot obtain a positive answer to the question of connection between the Quadrantid and $\delta$ Aquarid streams during the 4000 -yr interval. The orbital changes caused by the Poynting-Robertson effect will be negligible for the meteors discussed and can be disregarded.

\section{References}

Hamid, S. E. and Youssef, M. N.: 1963, Smithsonian Contr. Astrophys. 7, 309. Jacchia, L. G. and Whipple, F. L.: 1961, Smithsonian Contr. Astrophys. 4, 97. Shelton, J. W.: 1965, Astron. J. 70, 337. 